\title{
Fatores comportamentais desenvolvidos na adolescência e sua influência na etiologia do câncer de cavidade oral
}

\author{
Behavioral factors developed in adolescence and its influence on oral cavity cancer \\ etiology
}

Lidiane de Jesus Lisboa ${ }^{*}$, Marília de Matos Amorim², Sarah dos Santos Conceição², Alessandra Laís Pinho Valente Pires ${ }^{4}$, Igor Ferreira Borba de Almeida ${ }^{3}$, Maria Cristina Teixeira Cangussu4, Valéria Souza Freitas ${ }^{5}$

${ }^{1}$ Doutoranda em Saúde Coletiva pela Universidade Estadual de Feira de Santana (UEFS); ${ }^{2}$ Doutorandos em Saúde Coletiva pela Universidade Estadual de Feira de Santana (UEFS); ${ }^{3}$ Doutora em Saúde Pública pela Universidade de São Paulo (USP), Professora Associada da UFBA; ${ }^{4}$ Doutora em Ciências Odontológicas pela Universidade Federal do Rio Grande do Norte (UFRN), Professora Adjunto da UEFS

\begin{abstract}
Resumo
Introdução: o sistema de saúde brasileiro enfrenta um importante desafio com o aumento da incidência e taxa de letalidade das doenças crônicas não transmissíveis. Entre elas encontra-se o câncer de cavidade oral que se desenvolve com a interação de múltiplos fatores, especialmente de ordem ambiental, evitáveis, que são instalados muitas vezes durante a adolescência. Objetivo: realizar revisão de literatura referente à prevalência, em adolescentes, de fatores comportamentais de risco que podem levar ao desenvolvimento do câncer oral. Metodologia: foi realizada revisão narrativa a fim de levantar os principais trabalhos referentes à temática. O levantamento bibliográfico foi conduzido através de bases de dados on-line, referente ao período de 2008 a 2018, utilizando os descritores: fatores de risco comportamentais, adolescentes, câncer oral, tabaco, álcool, HPV, e dieta de forma combinada, nos idiomas português e inglês. Foram incluídos aqueles documentos que apresentaram as expressões utilizadas nas buscas no título ou palavras-chave, ou tinham explícito no resumo que o texto estava relacionado ao tema, dentro da temporalidade especificada. Resultados e Discussão: foram identificados 12 artigos sobre o tema que apontaram como principais fatores comportamentais desenvolvidos na adolescência o tabagismo, o alcoolismo, e os comportamentos sexuais e alimentares, os quais representam importantes fatores de riscos na instalação do câncer oral. Conclusão: é consenso na literatura a necessidade, na maioria dos casos, de acumulação temporal dos fatores etiológicos desta doença, tendo assim a adolescência como uma fase promissora para o estabelecimento de hábitos saudáveis ou nocivos para a saúde do indivíduo.
\end{abstract}

Palavras-chave: Adolescentes. Câncer oral. Fatores de risco.

\begin{abstract}
Introduction: the Brazilian health system faces an important challenge with the increasing incidence and case fatality rate of c chronic diseases. These include oral cavity cancer that develops through the interaction of multiple, especially environmental, preventable factors that often develop during adolescence. Objective: to review the literature regarding the prevalence in adolescents of behavioral risk factors that may lead to the development of oral cancer. Metodology: narrative review was performed in order to survey the main works related to the theme. The literature review was conducted through online databases, from 2008 to 2018, using the descriptors: behavioral risk factors, adolescents, oral cancer, tobacco, alcohol, HPV, and diet in a combined manner, in Portuguese and English. We included those documents that presented the expressions used in the search in the title or keywords, or had explicit in the abstract that the text was related to the theme, within the specified temporality. Results and Discussion: twelve articles on the topic were identified that pointed as the main behavioral factors developed in adolescence, smoking, alcoholism, and sexual and eating behaviors, which represent important risk factors in the onset of oral cancer. Conclusion: there is a consensus in the literature on the need, in most cases, for the temporal accumulation of the etiological factors of this disease, thus having adolescence as a promising phase for the establishment of healthy or harmful habits for the individual's health.
\end{abstract}

Keywords: Adolescents. Oral cancer. Risk factors.

\section{INTRODUÇÃO}

As transições demográficas e epidemiológicas observadas nas últimas décadas têm sido relacionadas ao aumento progressivo das doenças crônicas não transmissíveis (DCNT), dentre elas, o câncer, que é reconhecido

Correspondente/Corresponding: *Lidiane de Jesus Lisboa - Núcleo de Câncer Oral da Universidade Estadual de Feira de Santana, Prédio de Pós-Graduação em Saúde Coletiva. - End: Av. Transnordestina, Novo Horizonte, S/N, CEP: 44.036-900. Feira de Santana - Tel: (75) 3161-8096 -E-mail: lidianej.lisboa@gmail.com como um problema de saúde pública no Brasil, devido ao aumento da sua incidência e taxa de letalidade, representando um desafio para o sistema de saúde (FERLAY et al., 2013; OLIVEIRA; SOARES, 2002).

Com base nisso, o Instituto Nacional do Câncer (INCA) estima para o ano de 2019, na população brasileira a ocorrência de 600 mil casos novos de câncer, sendo que 14.700 serão de câncer de cavidade oral (INCA, 2017).

É importante destacar que o câncer é de etiologia multifatorial e se desenvolve de forma similar a outras do- 
enças, como resultado da interação de múltiplos fatores, genéticos e ambientais, especialmente de ordem comportamental, os quais colocam a população em contato com diferentes fatores de risco, a exemplo do tabagismo, o uso abusivo de bebidas alcoólicas, a alimentação inadequada, exposição a determinados vírus e outros fatores nocivos à saúde, muitas vezes, iniciados na adolescência (BARBOSA; CAMPOS, 2012; PETERSEN, 2009).

Embora a faixa etária mais acometida pelo câncer oral esteja acima dos 40 anos, o período da adolescência e adultos jovens compreende uma fase de alta vulnerabilidade devido às mudanças próprias da idade, e adoção de novas práticas e comportamentos, mas também, à exposição a diversas situações que envolvem riscos presentes e futuros para a saúde (CIRINO; NICHIATA; BORGES, 2010; RIBEIRO et al., 2015).

A adolescência é caracterizada como um período de grandes transformações físicas, emocionais, psíquicas e sociais que levam o adolescente a experimentar diferentes situações. Com isso, percebe-se o risco da aquisição de comportamentos influenciados pelo grupo, muitas vezes contraditórios, que expõem o jovem a potenciais riscos a sua saúde (DIAS; SAITER; CUNHA, 2015).

Diante desse cenário, onde alguns fatores relacionados ao câncer da cavidade oral são de ordem comportamental, o conhecimento sobre a instalação precoce destes hábitos na adolescência representa um importante desafio na área de saúde pública para o estabelecimento de políticas públicas para a prevenção da doença. Nesse sentido, o objetivo principal deste estudo é fazer uma revisão na literatura sobre a prevalência, em adolescentes, dos principais fatores comportamentais de risco para o câncer de cavidade oral.

\section{METODOLOGIA}

Realizou-se uma pesquisa do tipo revisão narrativa da literatura na qual buscou-se levantar os principais trabalhos referentes ao tema, sem, porém, pretender esgotar todas as fontes de informação.

Foram recuperados artigos indexados nas bases de dados on-line do LILACS, PubMed e SCIELO, tomando como referência o período de 2008 a 2018, empregando-se, de forma combinada, os descritores em inglês e português por meio da seguinte estratégia de busca: (fatores de risco comportamentais OR behavioral risk factors) AND (adolescentes OR teenagers) AND (câncer oral OR oral cancer) AND (tabaco OR tobacco) AND (álcool OR alcohol) AND (HPV OR Human papiloma virus) AND (dieta OR diet). Também foram feitas buscas a partir das referências bibliográficas dos trabalhos já selecionados e de buscas na internet. Os documentos incluídos nesta revisão foram selecionados por apresentarem as expressões utilizadas nas buscas no título ou palavras-chave, ou ter explícito no resumo que o texto tem pertinência ao tema, além de terem sido publicados nos últimos dez anos. Os critérios de exclusão envolveram publicações indisponíveis em formato completo, artigos que apresentassem relato de caso, monografias, teses e dissertações.

Utilizando os descritores propostos e após a leitura criteriosa dos títulos, palavras-chave e resumos, foram inicialmente selecionadas 27 publicações. De posse dos artigos completos e após a aplicação dos critérios de inclusão/exclusão estabelecidos para a revisão, foram selecionados 12 artigos.

\section{RESULTADOS E DISCUSSÃO}

Os artigos selecionados foram agrupados no (Quadro 1) de acordo com os fatores comportamentais que apresentaram.

Quadro 1-Frequência dos fatores comportamentais apontados nos artigos incluídos nesse estudo.

\begin{tabular}{|l|c|l|}
\hline $\begin{array}{c}\text { FATOR } \\
\text { COMPORTAMENTAL }\end{array}$ & FREQUÊNCIA & \multicolumn{1}{|c|}{ AUTOR/ANO } \\
\hline Tabagismo & 3 & $\begin{array}{l}\text { Petti (2009); Batista et al. } \\
\text { (2011); Currie et al. (2012). }\end{array}$ \\
\hline Etilismo & 3 & $\begin{array}{l}\text { Currie et al. (2012); Sculy, } \\
\text { Bagann (2009); Mccambridge, } \\
\text { Mcalaney, Rowe (2011). }\end{array}$ \\
\hline $\begin{array}{l}\text { Comportamento } \\
\text { Sexual }\end{array}$ & 3 & $\begin{array}{l}\text { Cirino, Nichiata, Borges (2010); } \\
\text { Chaturvedi et al. (2011); Young } \\
\text { (2015). }\end{array}$ \\
\hline $\begin{array}{l}\text { Comportamento } \\
\text { Alimentar }\end{array}$ & 6 & $\begin{array}{l}\text { Petti (2009); Currie et al. (2012); } \\
\text { Petersen (2009); Turati et al. } \\
\text { (2015); Marques et al. (2009); } \\
\text { Edefonti et al. (2012). }\end{array}$ \\
\hline
\end{tabular}

Fonte: Dados da pesquisa

O estilo de vida adotado na juventude pode ter consequências adversas na vida adulta, com o desenvolvimento das DCNT devido aos efeitos cumulativos que as práticas e comportamentos adotados possuem. Identificando assim, nesse grupo etário, a prevalência de hábitos de vida não saudáveis (LE-HÁ et al., 2013; RAPHAELLI; PRETTO; DUTRA, 2016).

A exposição a diversos fatores de risco comportamentais, como tabagismo, consumo de bebidas alcóolicas, alimentação inadequada e prática de sexo inseguro, tem, com frequência, início na adolescência, e estão associados ao desenvolvimento da maioria das DCNT, como as cardiovasculares, diabetes e câncer, principais causas de óbito na vida adulta no Brasil e no mundo (INCA, 2011; OLIVEIRA et al., 2013).

Dentre os fatores de risco comportamentais, destacam-se, o uso do tabaco e o consumo de bebidas alcoólicas, que continuam sendo descritos como os principais fatores associados a $80 \%$ dos casos de câncer oral no mundo ocidental (PETTI, 2009). Segundo Batista et al. (2011), o tabaco é o líder nas causas de mortes passíveis de prevenção no mundo. Além disso, o início do tabagismo em idade precoce está associado ao aumento da chance de uso de outras substâncias, como o consumo de bebidas alcoólicas e drogas ilícitas (CURRIE et al., 2012). 
De acordo com o INCA quanto mais precoce a idade do início do uso do tabaco, maior a probabilidade de dependência e consequentemente maior o risco de desenvolver o câncer (INCA, 2013).

Dados da Pesquisa Nacional de Saúde do Escolar (PeNSE), onde foram entrevistados 109.104 escolares do

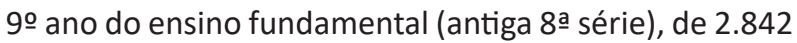
escolas públicas e privadas de todo o território brasileiro, revelaram que entre estudantes do 9ㅇ ano do ensino fundamental, a experimentação do cigarro foi de 19,6\%, sendo a maior frequência de experimentação observada na Região Sul $(28,6 \%)$ e a menor, na Região Nordeste $(14,9 \%)$ (INCA, 2012).

Outro estudo utilizando dados secundários, provenientes de um inquérito realizado em capitais do sul do Brasil entre 2002 e 2004, em escolas públicas e privadas, concluiu que a prevalência do tabagismo entre os escolares residentes nestas capitais é elevada e os fatores associados significativamente ao tabagismo era ter indivíduos fumantes entre os amigos e estar exposto à fumaça ambiental do tabaco fora de casa (HALLAL et al., 2009).

O consumo de bebida alcoólica também é um dos principais fatores de deterioração da saúde e está envolvido em mais de 60 diferentes causas de doenças, incluindo o câncer da cavidade oral, funcionando como potencializador do efeito do tabaco (SCULY; BAGANN, 2009).

A experimentação da bebida alcoólica também foi avaliada na PeNSE indicando que $66,6 \%$ dos escolares já haviam testado bebidas alcoólicas, sendo esse consumo maior nas Regiões Sul (76,9\%) e Centro-Oeste $(69,8 \%)$ e menor nas Regiões Norte (58,5\%) e Nordeste $(59,6 \%)$ (INCA, 2012). Além disso, o consumo excessivo de bebidas alcoólicas na adolescência está relacionado a outros problemas como insucesso escolar, acidentes, violências e outros comportamentos de risco, como tabagismo, uso de drogas ilícitas e sexo desprotegido (CURRIE et al., 2012).

Estudos realizados no Brasil e no mundo mostraram que a vida sexual dos adolescentes tem início cada vez mais cedo e que a precocidade está associada ao sexo desprotegido e ao maior número de parceiros ao longo da vida (CIRINO; NICHIATA; BORGES, 2010; SHAFII; STOVEL; HOLMES, 2007). A relação sexual precoce, o não uso de preservativos ou seu uso inadequado podem acarretar problemas como Infecções Sexualmente Transmissíveis (ISTs), HIV, gravidez indesejada e exposição a vírus relacionados à etiologia do câncer de colo de útero e cavidade oral, que pela imaturidade das genitálias favorece a sua instalação (CHATURVEDI et al., 2011; CONTI; BORTOLIN; KÜLKAMP, 2006).

Apesar da falta de consenso sobre a etiopatogênese do câncer de cavidade oral em adultos jovens, evidências sugerem uma relação da doença com o Herpes Papiloma Vírus (HPV), indicando que a infecção por este vírus possa ser uma possível explicação para o surgimento da doença nesses indivíduos, principalmente devido a mudanças no comportamento sexual que vem acontecendo ao longo dos últimos anos (CHATURVEDI et al., 2011). Evidências demostraram que um comportamento sexual durante toda a vida envolvendo um número mais elevado de parceiros com prática de sexo oral (maior que cinco parceiros) e vaginal (maior que 25 parceiros) estão associados ao aumento do risco de infecção por HPV nos casos de cânceres da região de cabeça e pescoço (YOUNG, 2015).

Outro provável fator de risco associado ao câncer oral é o hábito alimentar que é formado de modo gradual ao longo da vida, ocorrendo principalmente durante a primeira infância (INCA, 2011). Hábitos inadequados na infância e na adolescência podem ser fatores de risco para DCNT na idade adulta e em relação ao câncer, verifica-se que, depois do controle do tabaco, modificações saudáveis na dieta é a segunda maneira mais eficiente de prevenir esta doença (BARRETO et al., 2005).

Entre os adolescentes provenientes de famílias menos favorecidas, o consumo de alimentos como o arroz e o feijão são mais frequentes (VEIGA; SICHIERI, 2006). Ainda no que se refere ao padrão de consumo alimentar dos adolescentes, estudos internacionais confirmam o que tem sido apontado pela Organização Mundial de Saúde (OMS), a qual preconiza que é importante desenvolver hábitos de alimentação saudável entre crianças e adolescentes para sua manutenção na vida adulta e consequente redução de risco de doenças crônicas e obesidade (CURRIE et al., 2012). Dentre os hábitos considerados saudáveis, destaca-se o consumo de frutas e hortaliças como potencial fator de proteção para excesso de peso, doenças cardiovasculares, diabetes tipo 2 (CURRIE et al., 2012) e câncer, incluindo o de cavidade oral (PETERSEN, 2009; PETTI, 2009; TURATI et al., 2015).

Uma alimentação não saudável pode oferecer ao indivíduo substâncias carcinogênicas tais como os hidrocarbonetos aromáticos policíclicos presentes em alimentos cozidos ou processados em altas temperaturas, que podem ser encontrados nos embutidos (MARQUES et al., 2009). Com relação aos hábitos alimentares é sabido que uma alimentação altamente calórica e com grandes quantidades de gordura aumenta a chance de desenvolver câncer (BARRETO et al., 2005; EDEFONTI et al., 2012).

Dados da PeNSE realizada em 2012, também revelaram que o consumo de guloseimas (doces, balas, chocolates, chicletes, bombons ou pirulitos) em cinco dias ou mais na semana foi referido por $41,3 \%$ dos escolares. Em conjunto com o consumo de biscoitos salgados $(35,1 \%)$ e de refrigerantes (33,2\%), reafirmando as conclusões já observadas na PeNSE de 2009 acerca do padrão regular e elevado de consumo de alimentos não saudáveis por parcela significativa dos estudantes brasileiros (INCA, 2012).

A OMS destaca a necessidade de integração entre a saúde oral e geral, trazendo-as em unidade nas práticas de educação em saúde (PETERSEN; KWAN, 2004). Nestas ações é importante levar informações pertinentes à prevenção e sintomatologia das doenças, de modo a tornar a população participante, segura e empoderada para promover e prevenir a saúde (MELO et al., 2010). 


\section{CONCLUSÃO}

É consenso na literatura a necessidade, na maioria dos casos, de acumulação temporal dos fatores etiológicos desta neoplasia oral, tendo assim a adolescência como uma fase promissora para o estabelecimento de hábitos saudáveis ou nocivos para a saúde do indivíduo.

Os principais fatores comportamentais desenvolvidos na adolescência descritos nos artigos incluídos nessa revisão apontam o tabagismo, o alcoolismo, e os comportamentos sexuais e alimentares como possíveis atores envolvidos na instalação do câncer oral.

Com isso, esta revisão pode ser utilizada como subsídio para atualização dos profissionais de saúde nas ações de educação em saúde desenvolvidas na Atenção Primária à Saúde, além de direcionar o estabelecimento de políticas públicas para a prevenção do câncer de cavidade oral.

É importante que novos trabalhos sejam realizados visando estudar com mais profundidade esse tema, uma vez que limitações foram encontradas no desenvolvimento dessa pesquisa. Assim, estudos longitudinais que permitam confirmar essas associações são interessantes, principalmente para fomentar mudanças e melhorias nos hábitos de vida da população jovem.

\section{REFERÊNCIAS}

BARBOSA FILHO, V.C.; CAMPOS, W.; LOPES, A. S. Prevalence of alcohol and tobacco use among Brazilian adolescents: a systematic review. Revista de Saúde Pública, São Paulo, v. 46, p. 901-17, 2012.

BARRETO, S.M. et al. Análise da estratégia global para alimentação saudável, atividade física e saúde, da Organização Mundial da Saúde. Epidemiologia e Serviços de Saúde, Brasília, v. 14, n. 1, p. 41-68, 2005.

BATISTA E.S. et al. Impacto do Tabagismo e Álcool sobre a Composição Corporal de Jovens. Revista Brasileira de Cancerologia, Rio de Janeiro, v. 57, n. 3, p. 355-363, 2011.

BRASIL. INSTITUTO NACIONAL DE CÂNCER. Estimativa 2018: incidência de câncer no Brasil. Brasília: Instituto Nacional do Câncer, 2017. Disponível em: https://www.inca.gov.br/publicacoes/livros/estimativa2018-incidencia-de-cancer-no-brasil. Acesso em: 18 mar. 2018.

BRASIL. INSTITUTO NACIONAL DE CÂNCER. Programa Saber Saúde: prevenção do tabagismo e outros fatores de risco para o desenvolvimento de doenças crônicas não transmissíveis. Rio de Janeiro: Instituto Nacional do Câncer, 2013. Disponívele m: http:// bvsms.saude.gov.br/bvs/publicacoes/inca/Saber_saude_2013.pdf. Acesso em: 12 fev. 2017.

BRASIL. INSTITUTO BRASILEIRO DE GEOGRAFIA E ESTATÍSTICA. Pesquisa Nacional de Saúde do Escolar (PeNSE). Rio de Janeiro: Instituto Brasileiro de Geografia e Estatística, 2012. 256p. Disponível em: http://biblioteca. ibge.gov.br/visualizacao/livros/liv64436.pdf. Acesso em: 12 fev. 2017.

BRASIL. Ministério da Saúde e Ministério da Educação. Portaria Interministerial no 1.010, de 8 de maio de 2006. Institui as diretrizes para a promoção da alimentação saudável nas escolas de educação infantil, fundamental e nível médio das redes públicas e privadas, em âmbito nacional. Diário Oficial da União, Poder Executivo, Brasília, DF, n. 174, 9 set. 2011b. Seção 1, p. 21. Disponível em: http://bvsms.saude. gov.br/bvs/saudelegis/gm/2006/pri1010_08_05_2006.html. Acesso em: 12 fev. 2017
CHATURVEDI, A.K. et al. Human papillomavirus and rising oropharyngeal cancer incidence in the United States. J. Clin. Oncol., New York, v. 29, n. 32, p. 4294-4301, 2011.

CIRINO, F.M.S.B.; NICHIATA, L.Y.I.; BORGES, A.L.V. Conhecimento, atitude e práticas na prevenção do câncer de colo uterino e HPV em adolescentes. Escola Anna Nery Revista de Enfermagem, Rio de Janeiro, v. 14, n. 1 , p. $126-34,2010$.

CONTI, F. S.; BORTOLIN, S.; KÜLKAMP, I. C. Educação e promoção à saúde: comportamento e conhecimento de adolescentes de colégio público e particular em relação ao papilomavírus humano. DST - Jornal Brasileiro de Doenças Sexualmente Transmissíveis, Rio de Janeiro, v. 18, n. 1, p. 30-35, 2006.

CURRIE, C. et al. Social determinants of health and well-being among young people. Health Behaviour in School-aged Children (HBSC) study: international report from the 2009/2010 survey. WHO Regional Office for Europe, Copenhagen, v. 1, n. 6, p. 15-26, 2012

DIAS, A.; SAITER, M.; CUNHA, N. Avaliação de fatores de risco na adolescência. Revista Científica de Educação, São Paulo, v. 1, n. 1, p. 115-132, 2015.

EDEFONTI, V. et al. Nutrient-based dietary patterns and the risk of head and neck cancer: a pooled analysis in the International Head and Neck Cancer Epidemiology consortium. Annals of oncology, Oxford, v. 23, n. 7, p. 1869-1880, 2012.

FERLAY, J. et al. Cancer incidence and mortality worldwide: sources, methods and major patterns in GLOBOCAN 2012. International Journal of Cancer, Ohio, v. 136, n. 5, p. 359-386, 2015.

HALLAL, A. L. C. et al. Prevalência e fatores associados ao tabagismo em escolares da Região Sul do Brasil. Revista de Saúde Pública, São Paulo, v. 43, n. 5 , p. $779-88,2009$.

LE-HA, C. et al. Oral contraceptive use in girls and alcohol consumption in boys are associated with increased blood pressure in late adolescence. European Journal of Preventive Cardiology, Brailon, v. 20, n. 6, p. 947-55, 2013.

MARQUES, A. C.; VALENTE, T.B.; ROSA, C. S. Formação de toxinas durante $o$ processamento de alimentos e as possíveis consequências para o organismo humano. Revista de Nutrição, São Paulo, v. 22, n. 2, p. 283-293, 2009.

MCCAMBRIDGE, J.; MCALANEY, J.; ROWE, R. Adult consequences of late adolescent alcohol consumption: a systematic review of cohort studies. PLOS Medicine, San Francisco, v. 8, n. 2, p. 1-13, 2011.

MELO, C. et al. Perfil epidemiológico de casos incidentes de câncer de boca e faringe. Revista Gaúcha de Odontologia, Porto Alegre, v. 58, n. 3, p. 351-355, 2010.

OLIVEIRA, J. M. D. et al. Câncer de Boca: Avaliação do Conhecimento de Acadêmicos de Odontologia e Enfermagem quanto aos Fatores de Risco e Procedimentos de Diagnóstico. Revista Brasileira de Cancerologia, Rio de Janeiro, v. 59, n. 2, p. 211-218, 2013.

OLIVEIRA, M. N. G.; SOARES, E. A. Comparação do perfil dietético de adolescentes femininas e níveis socioeconômicos diferenciados. Nutrição Brasil, São Paulo, v. 1, n. 2, p. 68-76, 2002.

PETERSEN, E. P. Oral cancer prevention and control the approach of the World Health Organization. Oral Oncology, New York, v. 45, p.454460, 2009.

PETERSEN, P. E.; KWAN, S. Evaluation of community based oral health promotion and oral disease prevention - WHO recommendations for improved evidence in public health practice. Community Dental Health, New York, v. 21, p. 319-29, 2004. 
PETTI, S. Lifestyle risk factors for oral cancer. Oral Oncology, New York, v. 45, n. 4 , p. 340-350, 2009.

RAPHAELLI, C. O.; PRETTO, A. D. B.; DUtRA, G. F. Prevalência de hábitos de vida em escolares de um Município do Sul do Brasil. Revista Adolescência e Saúde, Rio de Janeiro, v. 13, n. 2, 2016.

RIBEIRO, I. L. A. et al. Fatores associados ao câncer de lábio e cavidade oral. Revista Brasileira de Epidemiologia, São Paulo, v. 18, n. 3, p. 618629, jul./set. 2015.

SCULLY, C.; BAGAN, J. Oral squamous cell carcinoma overview. Oral Oncology, New York, v. 45, n. 4, p. 301-308, 2009.

SHAFII, T.; STOVEL, K.; HOLMES, K. Association between Condom Use at Sexual Debut and Subsequent Sexual Trajectories: A Longitudinal Study Using Biomarkers. American Journal of Public Health, Washigton, DC, v. 97, p. 1090-1095, julho 2007.
TURATI, F. et al. Fruit and vegetables and cancer risk: a review of southern European studies. British Journal of Nutrition, Cambridge, v. 113, n. 2, p. 102-110, 2015.

VEIGA, G. V.; SICHIERI, R. Correlation in food intake between parents and adolescents depends on socioeconomic level. Nutrition Research, California, v. 26, n. 10, p. 517-523, 2006. Disponível em: http://www. sciencedirect.com/science/ article/pii/S027153170600193X. Acesso em: 13 mai. 2017.

YOUNG, D. Increase in head and neck cancer in younger patients due to human papillomavirus (HPV). Oral Oncology, New York, v. 51, n. 8, p. $727-730,2015$.

Submetido em: 01/09/2020

Aceito em: 15/10/2020 